\title{
Деградация параметров транзисторных датчиков температуры под действием ионизирующего облучения
}

\author{
(C) И.М. Викулин, В.Э. Горбачев , Ш.Д. Курмашев
}

Академия связи Украины, 65029 Одесса, Украина

ฯ E-mail: physonat@gmail.com

(Получена 26 декабря 2016 г. Принята к печати 27 декабря 2017 г.)

\begin{abstract}
Исследовано влияние эффективной концентрации задающей тип проводимости базовой области примеси и толщины базы на радиационную стойкость транзисторных термодатчиков. Получены зависимости прямого падения напряжения на эмиттерном переходе транзистора и коэффициента усиления по току от величины потоков электронов, нейтронов и $\gamma$-квантов. Получено, что деградация прямого падения напряжения под воздействием ионизирующего облучения начинается при дозах почти на 2 порядка выше, чем коэффициента усиления по току, в зависимости от конструктивных особенностей транзистора. После отжига облученных потоком электронов структур наблюдается значительное улучшение воспроизводимости термочувствительного параметра, что повышает процент выхода годных приборов.
\end{abstract}

DOI: $10.21883 /$ FTP.2017.10.45021.8447

\section{1. Введение}

Датчики температуры занимают особое место среди широкого разнообразия датчиков физических величин. Они работают на различных физических принципах, изготавливаются из различных материалов и позволяют измерять температуру в самых труднодоступных местах. Так, например, в активной зоне атомных реакторов преимущественно используют только датчики температуры. Показания их позволяют оценивать другие теплоэнергетические параметры (давление, плотность, уровень теплоносителя и др.). Наиболее перспективными для использования в качестве датчиков температуры являются полупроводниковые диоды и транзисторы, микросхемы и гибридные микросборки на их основе [1-3].

В качестве чувствительного параметра полупроводникового датчика температуры удобно использовать прямое напряжение на $p-n$-переходе. Основным преимуществом такого выбора является линейность температурной зависимости прямого напряжения на $p-n$-переходе в широком диапазоне рабочих температур. Это можно объяснить тем, что сопротивление $p-n$-перехода при прямом смещении в $10^{4}-10^{5}$ раз меньше, чем при обратном, поэтому шунтирующее влияние поверхностных каналов утечки даже при сильных изменениях температуры оказывается незначительным. Однако параметры диодного датчика температуры зависят от сопротивления базы и рекомбинации неосновных носителей в базовой области.

Эти недостатки диодных датчиков температуры можно устранить, если в качестве чувствительного элемента использовать биполярный транзистор при двухполюсном включении, когда его база и коллектор замкнуты. При этом в качестве термочувствительного параметра используется прямое падение напряжения на эмиттерном $p-n$-переходе. Хотя при таком включении характеристики транзистора эквивалентны характеристикам диодов, однако их воспроизводимость выше, чем у диодов, поскольку они в меньшей степени зависят от влияния сопротивления базы и рекомбинации неосновных носителей в базовой области. Более того, в транзисторном датчике в качестве чувствительного к температуре параметра можно использовать также коэффициент усиления по току на низких и высоких частотах. Несмотря на необходимость измерения температуры в средах с высокой интенсивностью радиационных воздействий, практически отсутствуют работы по исследованию влияния радиации на термочувствительные свойства транзисторов.

Цель работы - исследовать зависимость прямого напряжения на эмиттерном $p$-n-переходе $V$ и коэффициента усиления по току $\beta$ от величины потока электронов, нейтронов и $\gamma$-квантов, оценить влияние толщины базы и эффективной концентрации задающей тип примеси в базовой области (концентрации основных носителей заряда) на радиационную стойкость планарноэпитаксиальных транзисторных термодатчиков, изучить влияние отжига облученных структур на восстановление термочувствительных параметров.

\section{2. Экспериментальные результаты}

Для экспериментальных исследований из партии $p-n-p$-транзисторов типа КТ3107, изготовленных по планарно-эпитаксиальной технологии, использовались образцы со следующими параметрами: поверхностное сопротивление эмиттерной области $-0.5 \mathrm{OM} / \mathrm{cm}^{2}$, глубина залегания коллекторного $p-n$-перехода $\sim 0.1$ мм. Поверхностное сопротивление базовой области для раз-

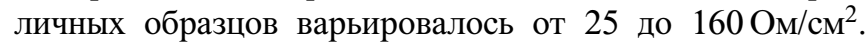
Были отобраны только те образцы, в которых толщина базы $W$ была либо 1.0, либо 2.1 мкм, а концентрация примеси в базе $N$ либо $7 \cdot 10^{17}$, либо $2.1 \cdot 10^{19} \mathrm{~cm}^{-3}$.

Исследуемые образцы были разделены на три группы. Транзисторы отдельной группы подвергались своему виду облучения: электронами, нейтронами или $\gamma$-квантами. 
Облучение проводилось в пассивном режиме. Напряжение на эмиттерном $p-n$-переходе $V$ и коэффициент усиления $\beta$ транзисторов измерялись до облучения и после каждого акта облучения заданной дозой. Поскольку транзисторные термодатчики работают при малых токах, то измерение $V$ проводили при токе коллектора $I_{\mathrm{c}}=1.2 \mathrm{MA}$, измерение $\beta$ проводили при напряжении на коллекторе $V_{\mathrm{c}}=2 \mathrm{~B}$, температура в момент измерений $T=293 \mathrm{~K}$.

Для изучения влияния $\beta$-излучения на исследуемые параметры транзисторы из первой группы были подвергнуты воздействию электронов с энергией $5 \mathrm{MэВ}$ четырех фиксированных значений потоков: $\Phi_{e}=10^{12}$, $10^{14}, 10^{15}, 5 \cdot 10^{15} \mathrm{~cm}^{-2}$. Облучение образцов производили на линейном ускорителе „Электроника“ ЭЛУ-4. Результаты измерений контролируемых параметров $V$ и $\beta$ представлены на рис. 1 и 2.

Эксперименты показали, что $V$ слабо меняется при увеличении потока электронов $\Phi_{e}$ практически во всем интервале его изменения. Уменьшение $V$ при потоках $\Phi_{e}>5 \cdot 10^{14} \mathrm{~cm}^{-2}$ характерно для термочувствительных структур с более тонкой базой (кривая 1 на рис. 1). После облучения максимальным для указанного диапазона потоком оно составляет около 5\% от первоначального значения, а для образцов с более толстой базой всего $3 \%$ (кривая 2 на рис. 1). Для образцов с большей концентрацией примеси в базе $N$ наблюдается усиление зависимости $V$ от величины потока электронов (кривая 3 на рис. 1), и уменьшение $V$ после облучения максимальным потоком будет порядка $8 \%$.

Таким образом, прямое напряжение на эмиттерном $p-n$-переходе термочувствительного транзистора подвержено меньшим изменениям при облучении электронами для образцов с толстой базой и меньшей концентрацией примеси в базе.

В отличие от $V$, изменение коэффициента усиления $\beta$ наблюдается уже при потоках $\Phi_{e}>5 \cdot 10^{12} \mathrm{~cm}^{-2}$. Резкое уменьшение коэффициента $\beta$ характерно для образцов с тонкой базой $W$ и малой концентрацией примеси в базе $N$ (кривая 1 на рис. 2). После облучения максимальным потоком оно составляет порядка $80 \%$ от первоначального значения. Для образцов с толстой базой (кривая 2 на рис. 2) и большой концентрацией примеси в базе (кривая 3 на рис. 2) изменения $\beta$ менее радикальны (около 70\%), но такие транзисторы до облучения имеют вдвое меньшее значение $\beta$.

Аналогичные изменения были получены для транзисторов из второй группы, которые подвергались воздействию $\gamma$-квантов с энергией $1.25 \mathrm{MэВ} \mathrm{шести} \mathrm{фикси-}$ рованных значений экспозиционных доз: $D_{\gamma}=10^{5}, 10^{6}$, $10^{7}, 10^{8}, 10^{9}, 10^{10} \mathrm{P}$. Облучение образцов в пассивном режиме производили на установке К-10000. В этом случае уменьшение напряжения $V$ на эмиттере датчика начинает проявляться после $\gamma$-облучения экспозиционными дозами $D_{\gamma}>10^{8} \mathrm{P}$. Причем, так же как и в первой группе, наибольшие изменения $V$ наблюдаются у транзисторов с тонкой базой и с большей концентрацией

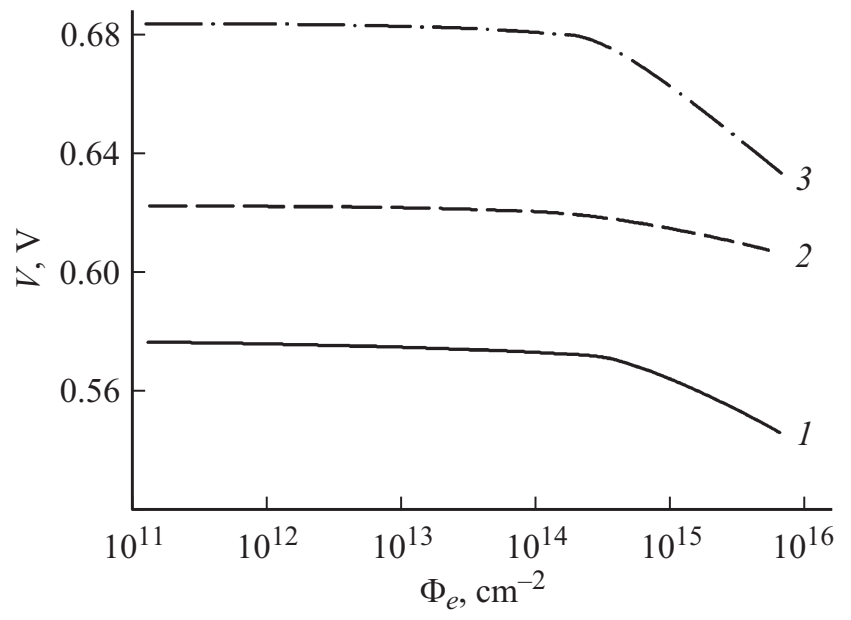

Рис. 1. Изменение напряжения на эмиттерном $p-n$-переходе транзистора после воздействия потока электронов. Толщина базы $W$, мкм: $1-1,2-2.1,3-1$. Концентрация примеси в базе до облучения $N, \mathrm{~cm}^{-3}: 1-7 \cdot 10^{17}, 2-7 \cdot 10^{17}$, $3-2.1 \cdot 10^{19}$

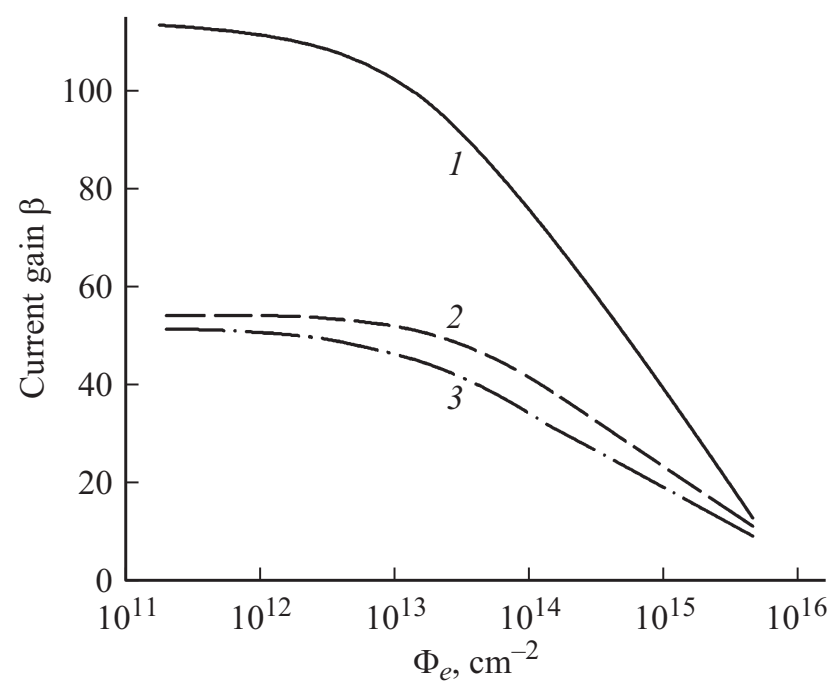

Рис. 2. Изменение коэффициента усиления термочувствительного транзистора после воздействия потока электронов. Толщина базы $W$, мкм: $1-1,2-2.1,3-1$. Концентрация примеси в базе до облучения $N, \mathrm{~cm}^{-3}: 1-7 \cdot 10^{17}, 2-7 \cdot 10^{17}$, $3-2.1 \cdot 10^{19}$.

примеси в базе. Деградация коэффициента усиления $\beta$ начинается после облучения значительно меньшими дозами $D_{\gamma}>10^{4} \mathrm{P}$. При этом образцы с толстой базой и большей концентрацией примеси в базе подвержены меньшим изменениям $\beta$ при облучении $\gamma$-квантами.

Похожие тенденции наблюдались в транзисторах из третьей группы, которые были подвергнуты воздействию нейтронов с энергией $14.5 \mathrm{MэB} \mathrm{четырех} \mathrm{фиксированных}$ значений потоков: $\Phi_{n}=10^{12}, 10^{13}, 10^{14}, 10^{15} \mathrm{~cm}^{-2}$. Источником быстрых нейтронов был генератор нейтронов типа НГ-150. Установлено, что только для образцов с тонкой базой и большей концентрацией примеси напряжение $V$ начинает незначительно уменьшаться лишь при 
облучении потоком $\Phi_{n}=10^{14} \mathrm{~cm}^{-2}$. У других образцов $V$ практически не изменялось во всем диапазоне исследований. Коэффициент усиления $\beta$ уменьшается уже при потоках $\Phi_{n}>10^{12} \mathrm{~cm}^{-2}$. При облучении образцов с тонкой базой и меньшей концентрацией примеси максимальным потоком его уменьшение было наибольшим и составляло $\sim 60 \%$ от первоначального значения.

Изменение термочувствительности $\Delta V / \Delta T$ после радиационного воздействия наблюдается лишь у транзисторов, у которых уменьшается $V$. Термочувствительность, как и напряжение $V$, деградирует сильнее в образцах с большей концентрацией примеси в базе. Однако, в отличие от напряжения $V$, для образцов с толстой базой уменьшение термочувствительности после облучения оказывается более значительным, чем для образцов с тонкой базой. Термочувствительность $\Delta V / \Delta T$ транзисторов с концентрацией примеси $N=2 \cdot 10^{19} \mathrm{~cm}^{-3}$ и базой $W=2.1$ мкм после облучения максимальным потоком электронов снижается на $8-10 \%$. У транзисторов с меньшей концентрацией примеси $N=7 \cdot 10^{17} \mathrm{~cm}^{-3}$ и тонкой базой $W=1$ мкм при указанных потоках изменения $\Delta V / \Delta T$ не наблюдается.

\section{3. Обсуждение экспериментальных данных}

Основным процессом образования радиационных дефектов в кремнии с относительно небольшой запрещенной зоной и значительной пороговой энергией дефектообразования является объемный механизм повреждения кристаллической структуры в результате смещения атомов. Первичными дефектами в кристаллах при радиационном воздействии являются вакансии и междоузельные атомы. В результате тепловой миграции первичных дефектов по кристаллу образуются различного рода комплексы: $A$ - и $E$-центры, дивакансии и др., которые создают в запрещенной зоне глубокие акцепторные уровни. При этом $E$-центры и дивакансии удаляют из зоны проводимости $n$-кремния по два электрона. Появление новых акцепторных уровней и захват вакансиями атомов донорной примеси резко уменьшает концентрацию основных носителей заряда в $n$-кремнии. Вместе с тем, время жизни неравновесных носителей заряда более чувствительно к появлению новых $A$-центров рекомбинации. Поскольку эффективное сечение захвата у таких генерационно-рекомбинационных уровней на 2 порядка больше, чем сечение прямой рекомбинации зона проводимости-валентная зона, поэтому деградация времени жизни неравновесных носителей заряда в кремнии начинается при меньших дозах облучения, чем деградация других параметров. Появившиеся в результате облучения комплексы в заряженном состоянии также являются дополнительными центрами рассеяния, которые уменьшают и подвижность носителей заряда. Однако деградация подвижности связана в основном с появлением $A$-центров рассеяния, поскольку образова- ние $E$-центра происходит одновременно с исчезновением донорного центра рассеяния.

Ухудшение электрических характеристик кремниевых приборов при облучении обусловлено главным образом эффектом удаления собственных носителей заряда и деградацией времени жизни неравновесных носителей заряда [4-6].

На рис. 3 сравниваются изменения эффективной концентрации основных носителей заряда, времени жизни неравновесных носителей заряда и их подвижности после радиационного облучения различными потоками. Наиболее быстро уменьшается концентрация основных носителей заряда (кривая 3). Однако такая деградация проявляется лишь при потоках $\Phi_{n} \sim 10^{14} \mathrm{~cm}^{-2}$, тогда как уменьшение времени жизни неравновесных носителей заряда уже сказывается при потоках $\Phi_{n} \sim 10^{11}-10^{12} \mathrm{~cm}^{-2}$ (кривые 1 и 2 ). Для больших значений времени жизни до облучения $\tau_{0}$ такая деградация начинается при меньших потоках облучения (кривая 1). Подвижность менее чувствительна к действию радиации, и ее уменьшение начинается при больших потоках облучения $\Phi_{n} \sim 10^{15} \mathrm{~cm}^{-2}$ (кривая 4 ).

Тенденции в деградации основных параметров полупроводника от потока радиационного облучения принято описывать эмпирическими соотношениями:

$$
\begin{gathered}
N=N_{0} \exp \left(-K_{N} \Phi\right), \\
\frac{1}{\tau}=\frac{1}{\tau_{0}}+K_{\tau} \Phi, \\
\frac{1}{\mu}=\frac{1}{\mu_{0}}+K_{\mu} \Phi,
\end{gathered}
$$

где $N_{0}, \tau_{0}, \mu_{0}-$ концентрация типозадающей примеси, время жизни дырок и их подвижность до облучения;

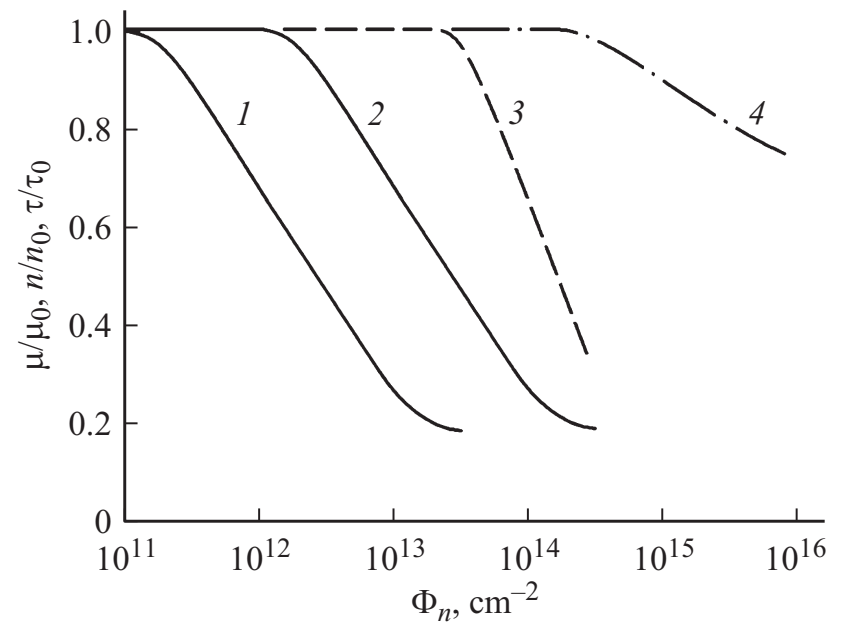

Рис. 3. Изменение относительных значений параметров кремния $n$-типа с удельным сопротивлением 1 Ом · см при облучении нейтронами с энергией $\sim 10$ кэВ: $1,2-$ времени жизни неравновесных носителей заряда $\tau / \tau_{0}, 3-$ концентрации основных носителей заряда $n / n_{0}, 4-$ подвижности $\mu / \mu_{0}$. Значение времени жизни до облучения $\tau_{0}$, с: $1-10^{-6}$, $2-10^{-9}$. 
$K_{N}, K_{\tau}, K_{\mu}-$ коэффициенты радиационного изменения концентрации типозадающей примеси, времени жизни неосновных носителей заряда и их подвижности; $\Phi-$ величина потока радиационного излучения. Коэффициенты $K_{N}, K_{\tau}, K_{\mu}$ зависят от параметров полупроводникового материала и различны для разных типов и диапазонов потоков радиационного воздействия [7]. Исследования показали, что время жизни неосновных носителей заряда в кремнии является параметром наиболее чувствительным к действию радиации, а деградацией подвижности, как эффектом 3-го порядка малости, по сравнению с изменением $N$ и $\tau$ можно пренебречь [7].

Прямое напряжение на эмиттерном выводе термочувствительного транзистора $V$ складывается из суммы напряжения непосредственно на $p-n$-переходе $V_{p-n}$ и падения напряжения на объеме базовой области $V_{\mathrm{b}}$ :

$$
V=V_{p-n}+V_{\mathrm{b}}
$$

Для тонкой базы биполярного транзистора при плотности прямого тока $J$ напряжения на $p-n$-переходе [1]

$$
V_{p-n}=\frac{k T}{q} \ln \frac{J W N}{q D_{p} n_{i}^{2}},
$$

где $W$ - толщина базы, $N$ - эффективная концентрация типозадающей примеси в базовой области (концентрация основных носителей), $D_{p}=k T \mu_{p} / q-$ коэффициент диффузии дырок, $\mu_{p}$ - подвижность дырок, $n_{i}-$ концентрация собственных носителей в базе.

Даже при небольших уровнях инжекции область базы вблизи $p-n$-перехода будет промодулирована инжектированными из эмиттера неравновесными носителями заряда, и падением напряжения на этой области в первом приближении можно пренебречь. Основной вклад в $V_{b}$ дает немодулированный неравновесными носителями остаток базы $\Delta W$, сопротивление которого определяется удельным сопротивлением материала базы $\rho_{\mathrm{b}}$ :

$$
V_{\mathrm{b}}=J \rho_{\mathrm{b}} \frac{\Delta W}{S}=\frac{J \Delta W}{S q \mu_{n} N},
$$

где $S$ - площадь $p-n$-перехода, $\mu_{n}-$ подвижность основных носителей в базе.

Как видно из соотношений (3) и (4), определяющим параметром при радиационном изменении обоих составляющих напряжения на датчике $V_{p-n}$ и $V_{\mathrm{b}}$ является концентрация типозадающей примеси в базе $N$. При этом, с учетом зависимости $N(\Phi)(1)$, получаем, что эффект уменьшения $V_{p-n}$ в результате облучения компенсируется эффектом увеличения $V_{b}$. Кроме того, анализ кривых, представленных на рис. 1, показывает, что деградация напряжения начинается при тех же потоках излучения, при которых начинает уменьшаться $N(\Phi)$ (рис. 3). Судя по полученной зависимости (кривая 1 на рис. 1), в транзисторах с узкой базой преобладает эффект уменьшения $V_{p-n}$. В транзисторах с большей шириной базы $W$ немодулированный неравновесными носителями участок базы $\Delta W$ будет больше, и падение напряжения на базе $V_{\mathrm{b}}(4)$ становится сравнимым с $V_{p-n}$, поэтому уменьшение $V_{p-n}$ частично компенсируется увеличением $V_{\mathrm{b}}$ после облучения (кривая 2 на рис. 1). Для заданной толщины базы можно подобрать такое значение тока, при котором оба эффекта компенсируются полностью, и напряжение на датчике не будет зависеть от действия радиации. В образцах с большой концентрацией примеси в базе $N$ вклад $V_{\mathrm{b}}(4)$ в общее напряжение $V$ уменьшится, а вклад $V_{p-n}(3)-$ увеличится, поэтому для таких образцов характерно более резкое уменьшение общего напряжения $V$ на датчике (кривая 3 на рис. 1) после радиационного воздействия.

Обратная величина коэффициента передачи тока транзистора определяется в основном рекомбинационными потерями в базе транзистора, в его эмиттере и на поверхности базы [1]:

$$
\frac{1}{\beta}=\frac{J_{\mathrm{b}}}{J_{k}} \approx \frac{W^{2}}{2 D_{p}^{\mathrm{b}} \tau_{p}^{\mathrm{b}}}+\frac{\rho_{e} W}{\rho_{\mathrm{b}} \sqrt{D_{p}^{e} \tau_{p}^{e}}}+\frac{s S_{\mathrm{sur}} W}{S D_{p}},
$$

где $\rho_{e}, \rho_{\mathrm{b}}$ - удельное сопротивление эмиттера и базы, $D_{p}^{e}$ и $\tau_{p}^{e}-$ коэффициент диффузии и время жизни дырок в эмиттере, $D_{p}^{\mathrm{b}}$ и $\tau_{p}^{\mathrm{b}}-$ коэффициент диффузии и время жизни дырок в базе, $s-$ скорость поверхностной рекомбинации, $S_{\text {sur }}$ - площадь поверхности, на которой происходит рекомбинация.

Как видно, определяющим параметром при радиационном изменении коэффициента передачи тока транзистора является время жизни дырок $\tau$. Поэтому деградация $\beta$ (рис. 2) начинается вместе с уменьшением $\tau$ (рис. 3) при значительно меньших потоках излучения, чем это наблюдается для $V$ (рис. 1), а резкий спад коэффициента $\beta$ при облучении объясняется сильной его зависимостью от $\tau(5)$. В образцах с более толстой базой и большей концентрацией примеси в базе увеличиваются и рекомбинационные потери, но в этом случае критическая плотность радиационных дефектов, при которой наблюдается резкое увеличение интенсивности рекомбинации, достигается при больших потоках радиации (кривые 2 и 3 на рис. 2).

Относительный вклад влияния $E$ - и $A$-центров на деградацию параметров $V$ и $\beta$ можно оценить по их восстановлению при нагреве (отжиге) облученных $\gamma$-квантами транзисторов. Как показали эксперименты, полное восстановление $V$ происходит при температурах $T>410 \mathrm{~K}$, тогда как для полного восстановления $\beta$ необходимо проводить отжиг при $T>620 \mathrm{~K}$. Эти данные позволяют утверждать, что, как мы предполагали выше, деградация прямого напряжения на эмиттерном $p-n$-переходе $V$ при радиационном воздействии происходит вследствие образования $E$-центров, отжиг которых происходит при температурах $400-420 \mathrm{~K}$, тогда как изменение коэффициента усиления $\beta$ обусловлено в основном образованием $A$-центров, температура отжига которых составляет $610-650 \mathrm{~K}$.

Следует отметить, что и концентрация задающей тип примеси в базе $N$, и время жизни носителей заряда $\tau$ 
одинаково резко изменяются при воздействии радиации (кривые 2 и 3 на рис. 3), при этом наблюдается относительно малое изменение напряжения $V$ после облучения (3-5\%) по сравнению с существенным изменением $\beta$ (70-80\%). Этот факт частично объясняется взаимной компенсацией двух вкладов в прямое напряжение на эмиттерном $p-n$-переходе $V(2)$ при облучении, которые, согласно (3) и (4), по-разному зависят от концентрации типозадающей примеси в базе. Однако если считать, что деградация напряжения $V$ связана в основном с образованием $E$-центров, которые, согласно относительно новым данным [8], формируют в запрещенной зоне не только акцепторные, но и донорные уровни, то необходимо учесть, что эти донорные состояния будут частично компенсировать уменьшение основных носителей в базе транзистора, вызванное появлением новых акцепторных уровней и захватом вакансиями атомов донорной примеси при образовании $E$-центра. Такая компенсация может достигать 60\% [9], что согласуется с данными наших экспериментов и объясняет малое изменение напряжения $V$ по сравнению с $\beta$.

Для изучения изменения термочувствительности при воздействии радиации выражение для напряжения на $p-n$-переходе (3) удобно представить в виде [1]

$$
V_{p-n}=\frac{E_{\mathrm{g}}}{q}-\frac{k T}{q} \ln \frac{c T^{4-\alpha}}{J W N},
$$

где $E_{\mathrm{g}}$ - ширина запрещенной зоны, $c-$ коэффициент, в который входят все, не зависящие от температуры, постоянные, $\alpha$ - показатель зависимости подвижности от температуры. Для уменьшения энергопотребления используется режим токов, при котором $J W N<c T^{4-\alpha}$, поэтому с ростом температуры напряжение на кремниевом $p-n$-переходе уменьшается на величину порядка $2 \mathrm{MB}$ на каждый градус. После облучения, когда абсолютное значение напряжения $V$ будет уменьшаться, снижается и термочувствительность датчика $\Delta V / \Delta T$. Для образцов с большей концентрацией примеси в базе $N$ до облучения второе слагаемое в (6) дает меньший вклад в общее напряжение, тогда и $\Delta V / \Delta T$, как и $V$, будет уменьшаться значительнее для тех же потоков облучения. В образцах с толстой базой этот вклад будет еще меньшим, а вклад напряжения на базе $V_{\mathrm{b}}(4)$ становится сравнимым с $V_{p-n}$. Радиационное воздействие увеличивает значение $V_{\mathrm{b}}$, что несколько уменьшает деградацию общего напряжения $V$, однако существенно ухудшает термочувствительность датчика, поскольку с ростом температуры напряжение на базе увеличивается, а $V_{p-n}$ уменьшается. Поэтому зависимость общего напряжения от температуры в образцах с более толстой базой в результате воздействия радиации ослабевает сильнее.

Анализ влияния облучения на термочувствительные транзисторы с различными конструктивно-технологическими параметрами показал, что деградация $\Delta V / \Delta T$ транзисторов с $W \geq 2.1$ мкм и поверхностным сопротивлением базы $R_{\mathrm{sb}} \leq 25 \mathrm{OM} / \mathrm{cm}^{2}$ начинается при меньших величинах воздействующих факторов, чем у образцов с меньшими $W$ и $R_{\mathrm{sb}}$. Следовательно, для повышения радиационной стойкости термочувствительных транзисторов необходимо уменьшать толщину базы и концентрацию примеси в базовой области.

Если рекомендация уменьшения $W$ носит общий характер, то условие уменьшения концентрации примеси $N$ для повышения радиационной стойкости не является безоговорочным для всех полупроводниковых приборов. По-видимому, существует оптимальное значение концентрации примеси в базовой области, обеспечивающее максимум на зависимости радиационной стойкости от $N$, т. е. наибольшую радиационную стойкость. Об этом свидетельствуют как результаты проведенных исследований, так и результаты исследований радиационной стойкости диодов с высокоомной базой. Оптимальное значение $N$ можно найти, если приравнять скорости изменения напряжения на $p-n$-переходе и падения напряжения на базовой области, которые, как известно, имеют разные знаки:

$$
\frac{\partial V_{p-n}}{\partial \Phi}=\frac{\partial V_{\mathrm{b}}}{\partial \Phi}
$$

Поскольку $V_{p-n}(3)$ и $V_{\mathrm{b}}(4)$ зависят также от $W$ и $J$, то данный экстремум является частным. Нахождение же главного экстремума является сложной задачей. Степень влияния облучения на параметры термочувствительных транзисторов при одинаковых значениях $N$ и $W$ зависит от градиента концентрации примеси в переходе. Этим и объясняется более высокая радиационная стойкость приборов, база которых сформирована диффузией бора, в отличие от приборов, база которых сформирована диффузией алюминия.

В работе [7] показано, что наибольшую радиационную стойкость имеют диоды с сильно легированной базой, т. е. низковольтные. Для повышения радиационной стойкости диодов рекомендуется делать диоды с тонкой базой и стараться, чтобы они работали при большом уровне инжекции, что задается режимом работы диода при прямом смещении. Однако для данного случая в этой рекомендации заложено некоторое противоречие: работа термодатчиков наиболее эффективна при низком уровне легирования базы и малом уровне инжекции. Таким образом, при обсуждении радиационной стойкости термодатчиков следует выбирать некоторый оптимальный вариант.

\section{4. Практическое применение результатов исследования}

Одним из основных требований, предъявляемых к технологии производства термочувствительных транзисторов, является обеспечение высокой воспроизводимости информационного параметра - падения напряжения на прямосмещенном эмиттерном $p-n$-переходе. На практике наблюдается значительный разброс этого параметра, что сказывается на проценте выхода годных приборов. 
Попытки улучшить воспроизводимость величины прямого падения напряжения с помощью известных методов - термотренировки и электротренировки оказались малоэффективными, так как позволяют в определенной мере лишь застабилизировать изменение термочувствительного параметра во времени. Экспериментально установлено, что облучение термочувствительных транзисторов потоком электронов $10^{14}-10^{15} \mathrm{~cm}^{-2}$ энергией 5-10 МэВ с последующим отжигом в течение 2-3ч позволяет существенно повысить воспроизводимость выходного параметра.

\section{5. Заключение}

В данной работе показано, что между прямым падением напряжения, коэффициентом усиления и термочувствительностью существует достаточно сильная корреляционная связь, которая нарушается при воздействии облучения. Деградация термочувствительных параметров (прямого падения напряжения) под воздействием облучения начинается при дозах на 1.5-2 порядка выше, чем коэффициента усиления. Степень деградации параметров термодатчика зависит от конструктивнотехнологических параметров транзисторов.

Эксперименты показали, что для обеспечения высокой радиационной стойкости термочувствительных транзисторов, действующих на основе зависимости $V$ от $T$, необходимо обеспечивать меньшие $N$ и $W$, т.е. обеспечивать те же требования, что и для получения максимальной термочувствительности.

\section{Список литературы}

[1] И.М. Викулин, В.И. Стафеев. Физика полупроводниковых приборов (М., Радио и связь, 1990).

[2] И.М. Викулин, Ш.Д. Курмашев, В.И. Стафеев. ФТП, 42, 113 (2008).

[3] В.М. Шарапов, Е.С. Полищук, Г.Г. Ишанин, А.Н. Гуржий, И.М. Викулин, Б.Н. Гордеев, Ю.Д. Жуков, Н.Д. Кошевой, Ш.Д. Курмашев, А.Н. Куценко, С.В. Марченко, И.Г. Минаев. Датчики (Киев, Брама, 2008).

[4] В.С. Вавилов, Н.П. Кекелидзе, Л.С. Смирнов. Действие излучений на полупроводники (М., Наука, 1988).

[5] V.V. Emtsev, P. Ehrhart, D.S. Poloskin, U. Dedek. Physica B: Condens. Matter, 273, 287 (2000).

[6] V.V. Emtsev, P. Ehrhart, D.S. Poloskin, K.V. Emtsev. J. Mater. Sci.: Mater Electron., 18, 711 (2007).

[7] Э.Н. Вологдин, А.П. Лысенко. Радиационные эффекты в некоторых классах полупроводниковых приборов (М., МГИЭМ, 2001).

[8] A. Nylandsted Larsen, A. Mesli, K. Bonde Nielsen, H. Kortegaard Nielsen, L. Dobaczewski, J. Abey, R. Jones, D.W. Palmer, P.R. Briddon, S. Oberg. Phys. Rev. Lett., 97, 106402 (2006).

[9] V.V. Emtsev, N.V. Abrosimov, V.V. Kozlovskii, G.A. Oganesyan. Semiconductors, 48, 1438 (2014).

\section{Degradation of parameters of transistor sensors of temperature under the action of ionizing irradiation}

\author{
I.M. Vikulin, V.E. Gorbachev, Sh.D. Kurmashev \\ Ukraine Academy of Communication, \\ 65029 Odessa, Ukraine
}

Abstract Influence of effective concentration of impurity, which defines of conductivity type in base region and thickness of base on the radiation resistance of transistor thermal sensors. The dependences of forward voltage on emitter junction and current amplification factor from magnitude of streams of electrons, neutrons and $\gamma$-quanta are obtained. A degradation of forward emitter bias after the ionizing irradiation begins when doses almost on two orders more, than for current amplification factor, depending on design features of the transistor, was obtained. After the annealing of the irradiated by stream of electrons structures the considerable enhancement of reproducibility of heat-sensitive parameter is observed, that raises percent of a yield of suitable devices.

Редактор А.Н. Смирнов 